\title{
A Comprehensive Nutriceutical Approach to Metabolic and Vascular Health
}

\author{
Frank H Comhaire* \\ Professor emeritus, Ghent University, Department of endocrinology and metabolic diseases, De Pintelaan, Belgium
}

Received: October 01, 2016; Accepted: October 26, 2016; Published: October 31, 2016

*Corresponding author: Frank H Comhaire, Professor emeritus, Ghent University, Department of endocrinology and Metabolic diseases, Brakelmeersstraat 18,9830 Sint Martens-Latem,Belgium, E-mail:frank@comhaire.com

\begin{abstract}
A composite nutriceutical containing extracts of Momordica charantia, pine bark extract, hematococcus pluvialis, red yeast rice, the antioxidants alpha-lipoic acid and oxidoreductase ubiquinone Q10, vitamins B9 and B12, and selenomethionine was given to 23 patients suffering from hyperinsulinemia and/or hyperlipemia as part of an open-label prospective cohort trial.

Treatment induced significant and persistent reduction of serum concentrations of insulin, C-peptide, glycated haemoglobin A1c, gamma-glutamyl transpeptidase, total and LDL cholesterol during 12 months of intake. Two patients interrupted treatment because of gastro-intestinal side effects. Vital parameters, renal function tests and triglycerids remained unchanged.

It is concluded that this composite nutriceutical improves biological markers and may reduce the risk of vascular pathology and non-alcoholic fatty liver disease. This premise needs to be confirmed by controlled clinical trials.
\end{abstract}

Keywords: Nutriceutical; Momordica charantia; Hyperinsulinemia; Atheromatosis; Cholesterol; Metabolic syndrome; Prediabetes; Monascus purpureus

\section{Introduction}

It has recently been proven that long-term low-dose multivitamin supplementation of healthy middle-aged men reduces the risk of vascular disease by $44 \%$ [1]. This could have been expected since resulting from - amongst other things antioxidant protection of the vascular wall and delayed oxidation of LDL-cholesterol [2]. At the other hand, there is increasing concern regarding the pandemic of obesity, which is associated with insulin resistance, the metabolic syndrome, diabetes [3], cardio- and cerebrovascular disease, and cancer. These induce massive financial burden on the health systems in many developed and developing countries $[4,5]$.

At present insulin resistance is commonly treated with Metformin, but long-term intake of this medication carries the risk of increased mortality among elderly patients suffering from impaired renal function [6], which is probably related to the accumulation of the drug causing lactic acidosis [7].
Hyperlipemia is successfully treated with statins, but a recent study has revealed that its long-term intake is associated with an important increase of the prevalence of diabetes [8] and enhances the glycated hemoglobin A1c, even in persons without diabetes [9].

Hence there is a need for complementary treatments for insulin resistance, the metabolic syndrome and hyperlipemia. The aim of this pilot open-label prospective cohort trial was to study the biological efficacy of a combined comprehensive nutriceutical that aims at reducing the latter conditions and that, eventually, may reduce the risk of vascular disease.

\section{Materials and Methods}

Patients coming to consultation at the private clinic of the author and presenting the biological characteristics of hyperinsulinemia [10] and/or hyperlipemia were offered the option to participate in a prospective, open-label, cohort trial using the composite nutriceutical food supplement described below. Patients suffered from either obesity and the metabolic syndrome, or type 2 diabetes. After having been extensively informed on the experimental character of the trial patients gave their informed consent and started treatment. Blood was taken 4 hours after dinner [11] at 1 month, and every 4 months afterwards in the same circumstances as before treatment. Blood analysis included measurement of glucose, hemoglobin A1c, insulin, C-peptide, total, low and high density lipoprotein cholesterol, triglycerids, gamma glutamyl transpeptidase, alanine aminotransferase, C-Reactive Protein (CRP), and creatinine. Measurements were performed using standardized methods by the laboratory Anacura (Evergem, Belgium) which was under continuous internal and external quality control.

Twenty three patients, 12 men (mean age 60.2 years, SD: 12.3 ) and 11 women (mean age: 53.8 years, SD: 16.8) have been included in this open label prospective cohort trial, of whom 7 have been followed for 12 months.

The nutriceutical was composed of the following substances: fermented red rice (Monascus purpureus), extract of bitter melon (Momordica charantia), alpha-lipoic acid, acetyl-l-carnitine, 
oxidoreductase ubiquinone Q10, pine bark extract, biomass of Haematococcus pluvialis, selenomethionine, Vit B9 (folic acid) and Vit B12 (cyanocobalamine)(Belgian patent 1021188). A proper dose of these components was processed into two capsules, and the patients were instructed to take two of each twice per day.

Data were collected in the spread sheet of medcalc statistical program (medcalc $₫$, Ostend, Belgium), and calculations included mean and standard deviation. Changes between data before treatment and during treatment were statistically analysed using the Wilcoxon's rank sum test for paired replicates, with P value $<0.05$ accepted as level of significance. Results are presented in dot and line plots or a histogram.

\section{Results}

There were no significant changes in the concentrations of C-reactive protein, creatinine, glucose and triglycerids. Insulin and C-peptide concentrations decreased significantly [figures 1 , 2] and so did the concentrations of hemoglobin A1c and gamma glutamyl transpeptidase [figures 3, 4]. Figure 5 shows the changes of mean values of the concentrations of cholesterol and lipoproteins.

\section{Discussion}

The nutriceuticals were well tolerated, except in 2 patients who interrupted treatment because of gastro-intestinal side effects.

Regular intake of the food supplement resulted in longterm suppression of both insulin and C-peptide concentrations measured 4 hours after dinner, indicating lower insulin resistance [12]. Improvement of the glucose metabolism also was evidenced by the permanently decreased glycated hemoglobin A1c concentration during treatment [13]. Thus, the present results confirm those published before on Momordica charantia

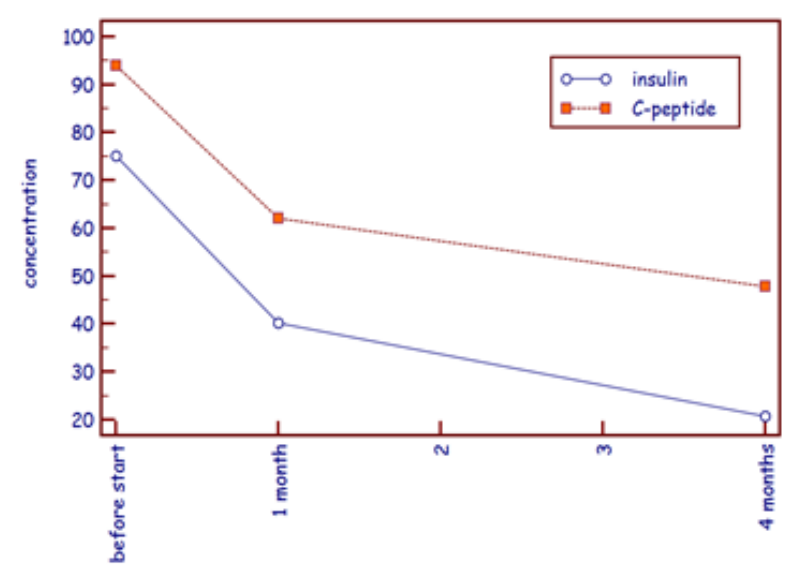

duration of treatment

Figure 1: Mean concentrations of insulin (on the vertical axis, in $\mu \mathrm{U} / \mathrm{L}$ ) and $\mathrm{C}$-peptide (on the vertical axis, in $\mathrm{ng} / \mathrm{mL}$ ) before treatment, and after 1 and 4 mths (on the horizontal axis, in months) of the nutriceutical intake.

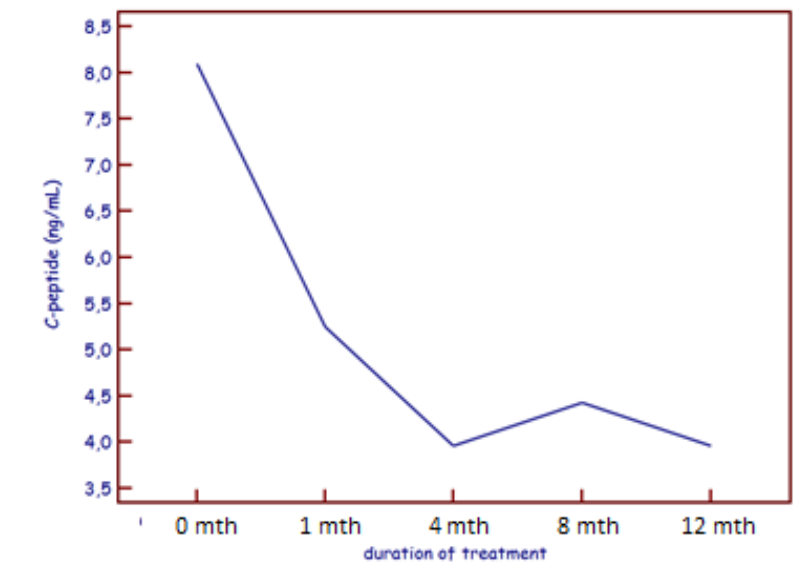

Figure 2: Mean concentration of C-peptide (on the vertical axis, in ng/ $\mathrm{mL}$ ) before treatment, and during 12 months (on the horizontal axis, in months) of nutriceutical intake in 7 patients.

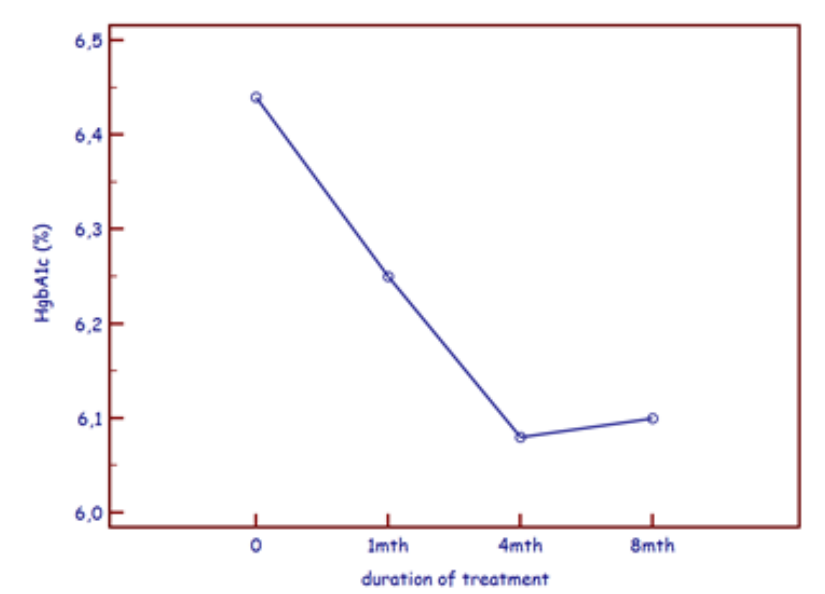

Figure 3: Mean concentration glycated hemoglobin A1c (on the vertical axis, in \%) before treatment, and during 8 months (on the horizontal axis, in months) of nutriceutical intake.

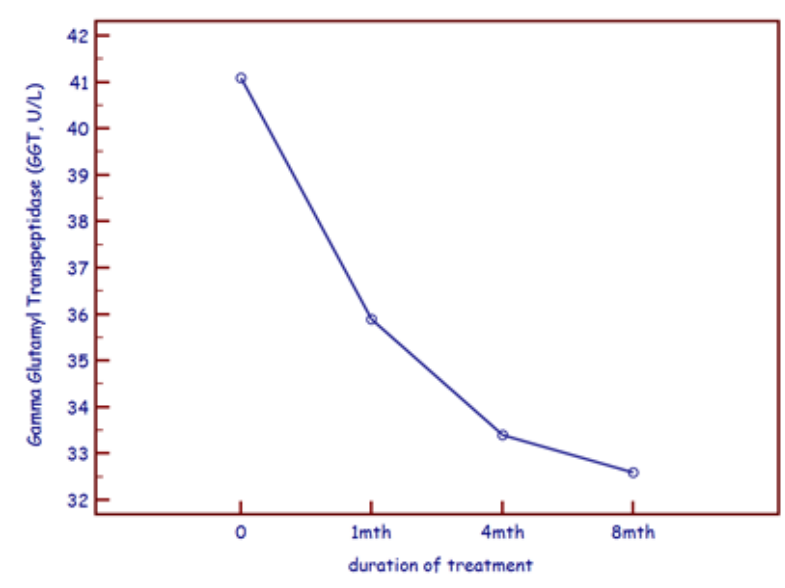

Figure 4: Activity of gamma glutamyl transpeptidase (on the vertical axis, in $\mathrm{U} / \mathrm{L}$ ) before treatment and during 8 months (on the horizontal axis, in months) of nutriceutical intake. 


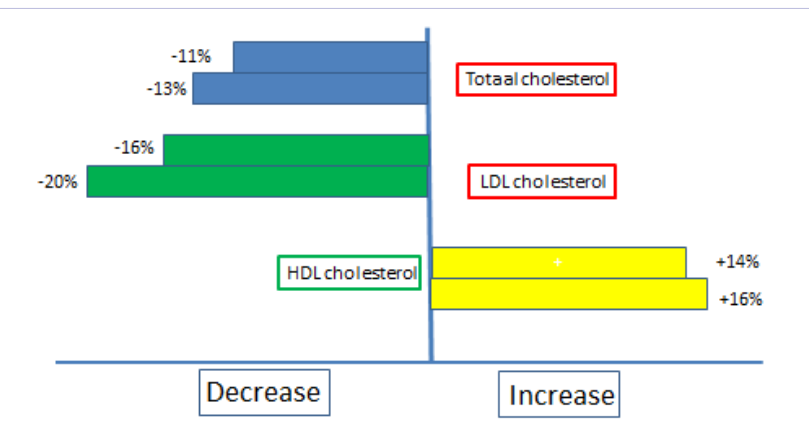

Figure 5: Mean changes (in \%compared to baseline) of total cholesterol, low density lipoprotein cholesterol (LDL cholesterol) and high density lipoprotein cholesterol (HDL cholesterol) after 1 month and after 3 months of nutriceutical intake.

[10], extending the duration of observation and increasing the number of participants.

Alpha-lipoic acid is an antioxidant that increases peripheral insulin sensitivity through different mechanisms, and has successfully been used in type 2 diabetic patients [14, 15].

Acetyl-l-carnitine is adequately absorbed after oral administration and enhances the transportation of fatty acids into the mitochondria [16] improving their function. Both in animals and humans carnitine supplementation was found to improve glucose tolerance [17], in particular during insulinresistant states [18].

The effect of Momordica charantia in reducing hyperisulinemia and improving metabolism was also evidenced by the significant decrease of the gamma glutamyl transpeptidase concentration, which is considered a marker of non-alcoholic fatty liver disease [19].

The components of the fermented red rice contributed to the cholesterol lowering effect and improved endothelial reactivity [20]. Quite remarkably, the ratio of total over HDL cholesterol was clearly reduced since HDL-cholesterol was increased, together with the rather strong decrease of LDL cholesterol, confirming the results of recent controlled trials [21, 22].

Since the monacolin present in fermented red rice also inhibits the synthesis of ubiquinone Q10 [23], this oxidoreductase was added to the nutriceutical formulation.

Astaxanthin in the biomass of Haematocoocus pluvialis exerts a strong antioxidant effect and delays the conversion of LDL cholesterol into its oxidized form [2].

Pine bark extract is rich in anthocyanidines reducing the activity of cyclo-oxygenase and of 5-lipoxygenase enzymes, which are of pivotal importance for atheromatous plaque formation [24].

Vitamins B9 and B12 reduce the concentration of homocysteïne, an independent risk factor of atheromatosis. These vitamins also decrease epigenetic DNA methylation through increasing the ratio of S-Adenosyl-Methionine (SAM) over S-Adenosyl Homocysteine (SAH) in the one carbon metabolism
[25].

Selenomethionine strengthens the favourable effect on the epigenome which is expected to decrease the risk of vascular disease [26].

Taken together these preliminary results suggest the comprehensive nutriceutical formulation may favourably influence the biological risk factors that are associated with vascular disease, by synergistically improving metabolic and epigenetic surrogate markers. However, the study presents major limitations due its short duration, the low number of subjects, its open-label design, and the investigation of biological variables only as end-point. Long-term controlled clinical trials are needed to sustain the premiss.

\section{Acknowledgement}

The author expresses his gratitude to pharmacist Johan Van Daele (Elversele, Belgium) for manufacturing the nutriceutical capsules.

\section{References}

1. Rautiainen S, Rist PM, Glynn RJ, Buring JE, Gaziano JM, Sesso HD. Mutivitamin use and the risk of cardiovascular disease. J Nutr. 2016. doi: 10.3945/jn.115.227884.

2. Bernard D, Christophe A, Delanghe J, Langlois M, De Buyzere M, Comhaire F. The effect of supplementation with an antioxidant preparation on LDL-oxidation is determined by haptoglobin polymorphism. Redox Rep. 2003;8:41-46. doi:http://dx.doi. org/10.1179/135100003125001233.

3. Editorial. Beat Diabetes: an urgent call for global action. The Lancet. 2016;387:1483. DOI: http://dx.doi.org/10.1016/S01406736(16)30185-4.

4. Quesenberry CP Jr, Caan B, Jacobson A. Obesity, health services use, and care cost among members of a health maintenance organization. Arch Intern Med. 1998;158(5):466-472.

5. Wolf AM, Colditz GA. Current estimates of the economic cost of obesity in the United States. Obes Res. 1998; 6(2): 97-106.

6. Hung SC, Chang YK, Liu JS, Kuo KL, Chen YH, Hsu CC, Tarng DC, et al. Metformin use and mortality in patients with advanced chronic kidney disease: national, retrospective, observational, cohort study. Lancet Diabetes Endocrinol. 2015;3(8):605-614. DOI: http://dx.doi. org/10.1016/S2213-8587(15)00123-0.

7. Boucaud-Maitre D, Ropers J, Porokhov B, Altman JJ, Bouhanick B, Doucet J, et al. Lactic acidosis: relationship between metformin levels, lactate concentration and mortality. 2016;33(11):1536-1543. doi: 10.1111/dme.13098.

8. Cederberg H, Stancakova A, Yaluri N, Modi S, Kuusisto J, Laakso M. Increased risk of diabetes with statin treatment is associated with impaired insulin sensitivity and insulin secretion: a 6 year follow-up study of the METSIM cohort. Diabetologia. 2015;58(5):1109-1117. doi: 10.1007/s00125-015-3528-5.

9. Ooba N, Tanaka S, Yasukawa Y, Yoshino N, Hayashi H, Hidaka S, et al. Effect of high-potency statins on HbA1c in patients with or without diabetes mellitus. J Pharm Health Care Sci . 2016 ;2:8 . doi: 10.1186/ s40780-016-0040-0.

10. Comhaire F. Treating hyperinsulinemia with Momordica charantia. J. 
Metab. Synd. 2015;4(3):1-5. doi:10.4172/2167-0943.1000177.

11. Nordestgaard BG, Langsted A, Mora S, et al. Fasting is not routinely required for determination of a lipid profile: clinical and laboratory implications including flagging at desirable concentration cut-points - a joint consensus statement from the European Atheroscerosis Society and European Federation of Clinical Chemistry and Laboratory Medicine. 2016. doi:10.1093/eurheartj/ehw152.

12. Jones AG, Hattersley AT. The clinical utility of C-peptide measurement in the care of patients with diabetes. Diabet Med. 2013;30(7):803817. doi: 10.1111/dme.12159.

13. Saudek CD, Brick JC. The clinical use of haemoglobin A1c. J Diabetes Sci Technol. 2009;3(4):629-634.

14. Kamenova P. Improvement of insulin sensitivity in patients with type 2 diabetes mellitus after oral administration of alpha-lipoic acid. Hormones (Athens). 2006;5(4):251-258.

15. Ansar H, Mazioom Z, Kazemi F, Hejazi N. Effect of alpha-lipoic acid on blood glucose, insulin resistance and glutathione peroxidase of type 2 diabetic patients. Saudi Med J. 2011;32(6):584-588.

16. Bremer.J. Carnitine-metabolism and functions. Physiol Rev. 1983;63(4):1420-1480.

17. Bloomer RJ, Fisher-Wellman KH, Tucker PS. Effect of oral acetyl L-carnitine arginate on resting and postprandial blood biomarkers in pre-diabetics. Nutr Metab (lond). 2009;6:25. doi: 10.1186/17437075-6-25.

18. Ringseis r, Keller J, Eder K. Role of carnitine in the regulation of glucose homeostasis and insulin sensitivity: evidence from in vivo and in vitro studies with carnitine supplementation and carnitine deficiency. 2012;51(1):1-18. doi: 10.1007/s00394-011-0284-2

19. Tahan V, Canbakan B, Balchi H, Dane F, Akin H, Can G, et al. Serum gamma-glutamyltranspeptidase distinguishes non-alcoholic fatty liver disease at high risk. Hepatogastroenterology. 2008; 55(85): 1433-1438.

20. Cicero AF, Morbini M, Rosticci M, D’Addato S, Grandi E, Borghi C. Middle-term dietary supplementation with yeast rice plus coenzyme Q10 improves lipid patter, endothelial reactivity and arterial stiffness in moderately hypercholesterolemic subjects. Ann Nutr Metab. 2016;68(3):213-219. doi: 10.1159/000445359.

21. Mazza A, Lenti S, Shiavon L, Zuin M, D'Avino M, Ramazzina E, et al. Nutraceutical for serum lipid and blood pressure control in hypertensive and hypercholesterolemic subjects at low cardiovascular risk. Adv Ther. 2015; 32(7): 680-690. doi: 10.1007/s12325-0150229-x.

22.Verhoeven V, Van der Auwera A, Van Gaal L, Remmen R, Apers S, Stalpaert M, Wens J, Hermans N. Can red yeast rice and olive extract improve lipid profile and cardiovascular risk in metabolic syndrome?: A double blind, placebo controlled randomized trial. BMC Complement Alter Med. 2015;15:25. doi: 10.1186/s12906-015-0576-9.

23. Yang HT, Lin SH, Huang SY, Chou HJ. Acute administration of red yeast rice (Monascus purpureus) depletes tissue coenzyme $Q(10)$ levels in ICR mice. Br J Nutr . 2005;93(4):131-135.

24. Ribeiro D, Freitas M, Tomé SM, Silva AM, Porto G, Cabrita E, et al. Inhibition of LOX by flavonoids: a structure-activity relationship study. Eur J Med Chem. 2014; 72: 137-145. doi: 10.1016/j. ejmech.2013.11.030.

25. Selhub J. Folate, vitamin B12 and vitamin B6 and one carbon metabolism. J Nutr Health Aginf . 2002;6(1):39-42.

26. Baccarelli A, Rienstra M, Benjamin EJ. Cardiovascular epigenetics: basic concepts and results from animal and human studies. CircCardiovasc Genet. 2010;3(6):567-573. doi: 10.1161/CIRCGENETICS.110.958744. 